NOTE UPON A FORM OF ACUTE INFLAMMATION OF THE CONJUNCTIVA ASSOCIATED WITH PUS COCCI.

BY SYDNEY STEPHENSON, M.B. EDIN., OPHTHALMIC SURGEON TO THE NORTH-EASTERN HOSPITAL FOR
CHILDREN, H'l'C.

IN children one now and then observes an acute inflammation of the conjanctiva along with a pustular eruption of the face or scalp, especially when in the form of eczema impetiginoides. ${ }^{1}$ It is thought that in these cases the con. unctiva has been inoculated by the contents of the fustules upon the skin, which are known to include staphylococci. The transference of contagious particles is brought about, as a rule, by the fingers of the patient, although in other instances purulent matter may run directly into the eye. I have examined bacteriologically several cases of this kind, and in all have succeeded in finding pyococci in the secretion from the inflamed conjunctiva. I am therefore inclined to think that the organisms named may, with fair probability, be looked upon as cansing the conjanctivitis. The following case is so typical of the entire series tbat it may seive to illustrate certain points with regard to diagnosis and treatment.

A female child, two years of age, was brought to the oph. thalmic department of the North-Eastern Hospital for Children on Sept. $15 \mathrm{th}, 1897$. The history was that a fortnight before "some yellow bumory heads" came out on the skin below the eyelids, especially on the left side of the face. Upon inquiry it was found that an elder sister had lately recovered from a similar eruption affecting the chin and mouth, probably impetiginous in nature. The patient's left eye had been inflamed for seven days. With the exception of weasles six months before coming under notice she had suffered no illness of any moment, and her eyes had been well in the interval. On examining the inflamed eye a considerable collection of yellowish-white discharge was seen in the lacus lacrymalis and the conjunctival sinuses. The lids were glossy and swollen, but not in the least tense; the eyeball was reddened; upon the upper and lower palpebral conjunctiva was a thin, greyish membrane, which could be readily detached, leaving the underlying tissues red and thick, with a slight tendency to bleed in a few scattered points. The cornea was bright and clear. The preauricular gland was not enlarged. The temperature of the patient was not raised; constitutional symptoms appeared to be absent. A few scabs were noticed upon the skin below the left lower eyelid. No discharge was present from the genitalia; there was no affection, as s welling or pain, of the joints. There was a negative history of sorethroat both as regards the child herself and those about her.

The diagnosis seemed to lie between an acute inflammation of the conjunctiva due to (1) Klebs-Löffler bacilli ; (2) gonococci ; (3) Weeks's bacilli ; (4) Fraenkel's pneumococci ; or (5) pyococci. From a clinical standpoint diphtheritis appeared to be excluded, since the eyelids could be separated without difficulty, the false membrane was easily detachable, the cornea was intact, the preauricular gland was not involved, there were no diphtheritic patches about the skin of the face, and constitutional symptoms were absent. Against purulent ophthalmia due to gonococci were the negative facts that the lids were not tense, that the secretion was not pus-like, that chemosis was not present, and, lastly, that dis sharge from the vulva or vagina was not present, as is apt to be the case among little girls. The unilateral character of the in flammation, although rather suggestive of pneumococcus infection, could not be regarded as telling much in any direction. Diagnosis, therefore, appeared to be narrowed down to an inflammation produced by Weeks's bacilli of catarrhal ophthalmia, by pneumococci, or by pus-organisms. The probability was that the micro-parasites last-named lay at the root of the mischief.

In order to determine this point a cover-glass preparation was made by lightly smearing a morsel of detached membrane over a glass slip, which was then stained in the usual why. The specimen, when examined with a one-twelfth oil immersion lens, showed merely a few scattered diplococci

1 Vide Dr. George Carpenter's paper on Pas Inoculations and Certain Eye Affections in Children, Archives of Pediatrics, vol, xi., 1894, p. 452. and cocci, lying upon and between the multinusleated and other cells contained in the preparation. These were found to retain their colour when treated according to Gram's method. This simple experiment was enough to yield valuable practical results, for in the first place it showed that diphtheria bacilli and Weeks's bacilli were absent; and secondly, that the organisms contained in the specimen must be either ordinary pus cocci or pneum0. cocci, inasmuch as gonococci, as is well known, are de. colourised by the alcohol employed in Gram's method. With a view to further identifying the organism or organisms known to be present two sloped agar-agar tubes were smeared with a morsel of membrane detached from the conjunctiva. and then placed in the incubator at $37.5^{\circ} \mathrm{C}$. Two days later each tube showed more or less discrete colonies of two kinds: (1) circular discs of orange hue from 1 to $3 \mathrm{~mm}$. in diameter; and (2) discs looking not unlike dabs of white oil paint from 0.25 to $2 \mathrm{~mm}$. in diameter. From each kind of colony cover-glass preparations were made, some being stained with weak carbolic-fuchsin and others by Gran's method. The colonies in all instances were found to consist of clustered cocci. Accordingly, the presumption was that one was dealing with the staphylococcus pyogenes aureus and albus-that is to say, with the organisms commonly found in styes, boils, carbuncles, and many other of the circumscribed suppurations. As a further proof, some of the water that had condensed in the original agar tubes was distributed in the usual way through three tubes of liquefied peptore-gelatin (10 per cent.), the contents of which were then poured into a similar number of flat-bottomed flasks like those used for the cultivation of bacteria in water. Colonies made their appearance within two days as minute masses visible to the naked eye. After five days' growth some of the colonies were observed to be white and others yellow in colour, while all the superficial ones lay, as it were, in little pits ufon the gelatin plate. Later, the medium became liquefied mure appreciably around individual colonies, which were found microscopically to respond to the well-known morphological and staining reactions of staphylococcus pyogenes albus and aureus.

As to treatment, a solation of silver nitrate, containing ton grains to the ounce, was applied to the everted conjanctivz of the inflamed eye every other day, and a wash of perchlo. ride of mercary ( 1 to 5000 ) used frequently. After ten applications of the silver there was scarcely any discharge from the conjunctiva, which was free from membrane, smooth, and but little swollen. The remedy was therefore discontinued. Upon Oct. 13sh the palpebral conjunctiva showed no obvious signs of disease, although some blepha. ritis, such as often follows an acute inflammation of the conjunctiva, was present. This was treated in the usual way, and the patient was discharged cured on 0ct. 27th.

As already stated, the above is typical of several cases is which I have found the two organisms named. This con. stant association of particular bacteria with a particular clinical type of conjunctivitis tends to incicate that the latter is caused by the former. In further support of this view the fact may be mentioned that upon two occasions 1 have seen an acute inflammation of the conjunctiva asso. ciated with otorrhœa, pus-organisms being present in the discharge both from the ear and from the eye.

Welbeck-street, $\mathrm{w}$.

\section{ON THE FLAGELLATED FORM OF THE MALARIAL PARASITE.}

By W. G. MACCALLUM, M.D., JOHNS HOPKINS HOSPTTAL, BALTIMORE.

Much attention has of late been devoted to the flagellate $Q$ forms of the malarial organism, and many theories as to its significance have been put forward, none of which have met with general credence. These-the theories of Laveran, Danilewsky, Mannaberg, Manson, Sakharoff, and the Italian school-are too well known to require repetition here. $15 y$ own observations on these forms as seen in the blood of crows, which are very generally infected with the halteridium of Labbé, have led me to quite different conclusions, and are briefly as follows.

Frequently in slides of the blood of infected crows there appear, after standing from twenty to thirty minates, elongated motile forms such as were described by Danilewskg as vermiculi in his "Parasitologie Comparée du Sang"; and 